\section{Público versus privado: avaliando a assistência à gestação e ao parto no extremo sul do Brasil}

\section{Public versus private: assessing maternity care in the far South of Brazil}

Juraci A. Cesar 1

Patrícia S. Mano 2

Kharen Carlotto 3

David A. Gonzalez-Chica 4

Raul A. Mendoza-Sassi 5

1,2,5 Divisão de População \& Saúde. Universidade Federal do Rio Grande. Rua General Osório s.n. $4^{\circ}$ andar. Centro. Campus Saúde Área Acadêmica Prof. Newton Azevedo. Rio Grande, RS, Brasil. CEP: 96.201-900. E-mail: juraci.cesar@gmail.com

3 Área de Saúde Materno-Infantil. Universidade Federal do Rio Grande. Rio Grande, RS, Brasil.

4 Departamento de Nutrição. Universidade Federal de Santa Catarina. Florianópolis, SC, Brasil.

\section{Resumo}

Objetivos: avaliar a assistência à gestação e ao parto entre o setor público e privado no municipio de Rio Grande, RS.

Métodos: aplicou-se questionário padronizado a todas as gestantes residentes neste municipio que tiveram filho em 2007. Investigaram-se aspectos relativos a cuidados recebidos desde o início da gestação até o pós-parto imediato. A análise estatística consistiu da comparação de proporções nestes dois grupos através do teste de quiquadrado.

Resultados: dentre os 2584 nascimentos cujas mães residiam no municipio, foram obtidas informações sobre 2557, o que representa 98,9\% do total. Destas mães, $96 \%$ realizaram pelo menos uma consulta de pré-natal. Gestantes atendidas no setor privado iniciaram o pré-natal mais cedo, realizaram um maior número de consultas médicas, exame de sangue e ultrassonografia pélvica, exame ginecológico, das mamas e citopatológico de colo uterino. Gestantes do setor público realizaram maior número de exames de urina e sorologia para sifilis e foram mais comumente suplementadas com sulfato ferroso. Todas estas diferenças foram estatisticamente significativas $(p<0,05)$.

Conclusões: gestantes do setor privado receberam de forma sistemática melhor assistência durante o pré-natal em termos de consultas e exames realizados, tiveram seu parto mais comumente realizado por médico, foram mais afetadas por intervenções desnecessárias como cesariana e episiotomia e menos frequentemente suplementadas com ferro.

Palavras-chave Cuidado pré-natal, Parto, Assistência à saúde, Cesária, Serviços de saúde 


\section{Introdução}

A realização do pré-natal adequado é essencial à redução da morbimortalidade materno-infantil. Estima-se que pelo menos $10 \%$ dos óbitos infantis poderiam ser evitados se todas as gestantes realizassem pelo menos seis consultas durante o prénatal e fossem submetidas a exames clínicos e laboratoriais básicos. Em algumas regiões do país, o impacto destas medidas poderia reduzir em até $25 \%$ o coeficiente de mortalidade infantil. ${ }^{1}$

No ano 2000, o Ministério da Saúde instituiu o Programa de Humanização no Pré-natal e Nascimento (PHPN). Esta iniciativa visava reduzir as altas taxas de morbimortalidade materna e perinatal, assegurar acesso, melhorar a cobertura e a qualidade do pré-natal, da assistência ao parto, puerpério e neonatal. Buscava ainda instituir postura ética e solidária por parte dos profissionais e fazer com que as unidades de saúde recebessem de forma acolhedora a mulher, familiares e o recém-nascido. Por fim, recomendava a adoção de procedimentos sabidamente benéficos à mulher e ao recém-nascido evitando práticas intervencionistas desnecessárias. ${ }^{2}$

Várias publicações asseguram que o pré-natal realizado no setor privado é melhor que o do setor público.3-5 Isto é feito comparando a prevalência de indicadores como, por exemplo, o percentual de gestantes que realizou pelo menos sete consultas durante o pré-natal ou que tiveram filho com peso menor que 2500 gramas (baixo peso ao nascer), entre estes dois setores, 5 ou ainda, utilizando-se de critérios que avaliam a adequação da qualidade do pré-natal recebido. Neste sentido, os critérios mais utilizados são os propostos por Coimbra et al.3,4 e por Takeda, 6 ambos baseados no número de consultas realizadas e na idade gestacional por ocasião do início do pré-natal. Outro critério menos utilizado, porém mais abrangente e mais apropriado, é o proposto por Silveira et al. ${ }^{7}$ Este critério inclui além do número de consultas e da idade gestacional de início, a realização de pelo menos dois exames qualitativos de urina, hemoglobina e Venereal Disease Research Laboratory (VDRL).

Este artigo tem por objetivo avaliar a qualidade do pré-natal recebido por gestantes atendidas nos setores público e privado a partir da comparação de indicadores isolados da assistência à gestação e ao parto e da aplicação do critério de avaliação da adequação do pré-natal proposto por Silveira et al.7 para todos os nascimentos ocorridos em um município da Região Sul do Brasil.

\section{Métodos}

O município de Rio Grande, localizado no Estado do Rio Grande do Sul, tem cerca de 200 mil habitantes, com $95 \%$ deles residindo em área urbana. $\mathrm{O}$ seu produto interno bruto (PIB) per capita/ano é de aproximadamente $\mathrm{R} \$ 19.000,00$ e no quesito desenvolvimento, ocupa a $29^{\mathrm{a}}$ posição dentre os 453 municípios do Estado; o coeficiente de mortalidade infantil vem declinando nos últimos anos, caiu de 23/1000 em 2003 para 15/1000 em 2007, mas ainda era superior a média do Estado, que apresentou média para 2007 de 13/1000. A rede básica de saúde no município era constituída por 32 unidades, com $85 \%$ delas tradicionais e as demais com a Estratégia Saúde da Família. Havia ainda dois hospitais gerais de média complexidade e cinco ambulatórios destinados ao atendimento de especialidades médicas. 8

Foram incluídos neste estudo todos os nascimentos ocorridos nas duas únicas maternidades do município entre $1^{\circ}$ de janeiro e 31 de dezembro de 2007. Para fazer parte do estudo, o recém-nascido deveria alcançar peso igual ou superior a 500 gramas ou pelo menos 22 semanas de idade gestacional e suas mães residirem no município eleito.

Realizou-se um estudo de delineamento transversal. As informações foram coletadas através de um único questionário pré-codificado que buscava as seguintes informações: local de residência da família (urbano ou rural); características demográficas e reprodutivas maternas; nível socioeconômico e condições de habitação da família; assistência à gestação e o parto: início da das consultas de prénatal; número de consultas e de exames clínicos e laboratoriais; medidas da criança: comprimento, perímetro cefálico e torácico e circunferência abdominal. O peso ao nascer foi anotado a partir do livro de registro da enfermagem, que utiliza nos dois hospitais, balança eletrônica pediátrica com precisão de $10 \mathrm{~g}$, com aferição semanal utilizando-se de pesos padrão. A idade gestacional dos nascidos vivos foi avaliada a partir da data da última menstruação (DUM) anotada no cartão da gestante. Considerouse como prematura a criança que nasceu com menos de 37 semanas de gestação, valor este obtido pela subtração da data de nascimento da DUM.

Para a avaliação da adequação do pré-natal foi utilizado o índice proposto por Silveira et al. ${ }^{7}$ Este índice considera o pré-natal como: adequado - realização de seis ou mais consultas; o início ocorrendo antes do quinto mês de gestação e a realização de dois ou mais exames qualitativos de urina, hemoglobina e VDRL; inadequado - realização de três consultas; o início após o sétimo mês de gestação e 
não realização de qualquer exame laboratorial do item anterior e, por fim, intermediário - para as demais situações. Dados não lembrados pelas gestantes foram classificados na categoria ignorado.

Considerou-se como tendo realizado o pré-natal no setor público ou privado a gestante que fez a maioria das consultas nas unidades básicas e nos ambulatórios da rede pública de saúde do município ou em clínicas e consultórios particulares, respectivamente. Em caso de realizar igual número de consultas nos dois setores, a definição era dada pelo local de realização da maioria das consultas no primeiro trimestre de gravidez e, se necessário, no segundo trimestre, consecutivamente.

Um estudo piloto foi realizado nas maternidades no mês imediatamente anterior ao início da coleta de dados. As mães pesquisadas foram entrevistadas na maternidade em até 24 horas após o parto, por dez entrevistadores previamente treinados para a pesquisa. Para o controle de qualidade, foram refeitas parcialmente cerca de $10 \%$ das entrevistas com as mães em suas residências.

Todas as questões abertas eram codificadas pelo entrevistador; os questionários foram duplamente digitados para verificar sua consistência; a entrada de dados foi realizada utilizando-se do programa Epi Info 6.04.9 Por fim, realizou-se análise de consistência através do pacote estatístico Stata versão 9.2.10
Cada uma das entrevistadas assinou um termo de consentimento livre e esclarecido antes da realização da entrevista. O protocolo de pesquisa foi submetido e aprovado pelo Comitê de Ética em Pesquisa na Área da Saúde (CEPAS) da Universidade Federal do Rio Grande (FURG).

\section{Resultados}

Segundo o Sistema de Informações de Nascidos Vivos (SINASC), em 2007 ocorreram 2584 nascimentos entre os residentes no município de Rio Grande. Destes, foram entrevistadas 2557 mães, o que representa $98,9 \%$ do total. Dos $34(1,1 \%)$ nascimentos restantes, uma mãe recusou-se a participar do estudo e as demais não foram localizadas.

A Tabela 1 mostra que somente duas em cada três gestantes do setor público realizaram seis ou mais consultas de pré-natal e iniciaram o pré-natal no primeiro trimestre contra cerca de nove em cada dez no setor privado. O número médio de consultas realizadas no setor público foi $6,6(\mathrm{DP}=2,9)$, enquanto no setor privado foi 9,6 (DP=3,5). Esta diferença foi menos evidente em relação à imunização antitetânica ( $84 \%$ vs $90 \%)$. Em relação aos exames laboratoriais, gestantes do setor público levaram ligeira vantagem na realização de dois ou mais exames de urina ( $37 \%$ vs $32 \%$ ) e sorologia para sífilis $(27 \%$ vs $23 \%)$, enquanto aquelas do setor

Tabela 1

\begin{tabular}{|c|c|c|c|}
\hline \multirow[t]{3}{*}{ Variável } & \multicolumn{2}{|c|}{ Setor de saúde } & \multirow[t]{3}{*}{$p$} \\
\hline & \multirow{2}{*}{$\frac{\text { Público }(\mathrm{N}=1493)}{\%}$} & \multirow{2}{*}{$\begin{array}{c}\text { Privado }(\mathrm{N}=954) \\
\%\end{array}$} & \\
\hline & & & \\
\hline Realizaram seis ou mais consultas de pré-natal & 65,5 & 90,6 & $<0,001$ \\
\hline Iniciaram o pré-natal no primeiro trimestre & 65,5 & 86,2 & 0,000 \\
\hline Imunização antitetânica completa & 84,4 & 90,4 & $<0,001$ \\
\hline \multicolumn{4}{|l|}{ Exames laboratoriais realizados duas vezes ou mais: } \\
\hline Urina & 36,7 & 32,3 & 0,029 \\
\hline Hemoglobina & 81,5 & 87,1 & 0,001 \\
\hline Anti-HIV & 70,1 & 70,8 & 0,722 \\
\hline VDRL & 26,9 & 22,8 & 0,029 \\
\hline Ultrassonografia pélvica obstétrica & 55,8 & 93,7 & 0,001 \\
\hline \multicolumn{4}{|l|}{ Exames clínicos realizados pelo menos uma vez: } \\
\hline Verificação da pressão arterial & 97,0 & 99,5 & $<0,001$ \\
\hline Pesagem & 98,9 & 96,9 & $<0,001$ \\
\hline Avaliação da altura uterina & 99,2 & 97,9 & 0,006 \\
\hline Exame ginecológico & 51,9 & 69,2 & $<0,001$ \\
\hline Exame das mamas & 40,2 & 57,4 & $<0,001$ \\
\hline Citopatológico de colo uterino & 57,5 & 69,4 & $<0,001$ \\
\hline Suplementação com sulfato ferroso & 67,5 & 52,5 & $<0,001$ \\
\hline
\end{tabular}

HIV= vírus da imunodeficiência adquirida; VDRL= Veneral Disease Research Laboratory. 
privado mostraram nítida vantagem na realização da dosagem de hemoglobina ( $87 \%$ vs $81 \%$ ) e ultrassonografia pélvica ( $94 \%$ vs 56\%). A realização de exame ginecológico, das mamas e citopatológico de colo uterino foram baixas, mas ainda com vantagem significativa para gestantes atendidas no setor privado. Por fim, a suplementação com sulfato ferroso cobriu cerca de dois terços das gestantes atendidas no setor público e pouco mais da metade no setor privado.

A Tabela 2 refere-se à assistência recebida durante o parto. Gestantes atendidas no setor privado tiveram acompanhamento na internação mais frequentemente acompanhada por médico (31\% vs $26 \%)$ assim como a realização do parto $(94 \%$ vs
$82 \%$ ) por este profissional; foram mais comumente submetidas à cesariana $(70 \%$ vs $43 \%)$ e episiotomia ( $81 \%$ vs $71 \%$ ); seus filhos nasceram com melhor índice de Apgar tanto no primeiro (93\% vs 90\%) quanto no quinto minuto ( $99 \%$ vs $98 \%$ ) e foram mais frequentemente atendidos por pediatra na sala de parto $(97 \%$ vs $92 \%)$. Para as variáveis "uso de fórcipe" ( $7 \%$ vs $8 \%)$ e média de "peso ao nascer" $(3210 \mathrm{~g}[\mathrm{DP}=609 \mathrm{~g}]$ vs $3168 \mathrm{~g}[\mathrm{DP}=526 \mathrm{~g}])$ não houve diferença significativa entre os dois grupos.

A avaliação da adequação do pré-natal a partir do critério proposto por Silveira et al. ${ }^{7}$ mostrou índice de adequação de $18 \%$ para ambos os grupos estudados (Tabela 3 ).

Tabela 2

Assistência ao parto conforme o setor de saúde utilizado. Rio Grande, RS, 2007.

\begin{tabular}{|c|c|c|c|}
\hline \multirow[t]{3}{*}{ Variável } & \multicolumn{2}{|c|}{ Setor de saúde } & \multirow[t]{3}{*}{$p$} \\
\hline & \multirow{2}{*}{$\begin{array}{c}\text { Público }(\mathrm{N}=1493) \\
\%\end{array}$} & \multirow{2}{*}{$\begin{array}{c}\text { Privado }(\mathrm{N}=954) \\
\%\end{array}$} & \\
\hline & & & \\
\hline \multicolumn{3}{|l|}{ Acompanhamento durante a internação } & 0,012 \\
\hline Médico & 26,0 & 31,3 & \\
\hline Estudante & 18,1 & 15,4 & \\
\hline Enfermeira/Parteira & 56,0 & 53,3 & \\
\hline Se o parto foi realizado por médico & 81,6 & 93,8 & $<0,001$ \\
\hline \multicolumn{3}{|l|}{ Tipo de parto } & $<0,001$ \\
\hline Normal & 57,4 & 30,2 & \\
\hline Cesariana & 42,6 & 69,8 & \\
\hline Foi necessário usar fórcipe & 7,8 & 7,3 & 0,761 \\
\hline Foram submetidas à episiotomia $(n=1238)$ & 70,9 & 81,3 & 0,001 \\
\hline \multicolumn{3}{|l|}{ Peso ao nascer $(\mathrm{g})$} & 0,087 \\
\hline$<2500$ & 9,7 & 7,2 & \\
\hline 2500 a 2999 & 23,5 & 22,1 & \\
\hline 3000 a 3499 & 39,4 & 43,0 & \\
\hline$\geq 3500$ & 27,3 & 27,7 & \\
\hline Natimorto & 1,9 & 0,5 & 0,005 \\
\hline \multicolumn{4}{|l|}{ Índice de Apgar } \\
\hline$\geq 7$ no primeiro minuto & 90,3 & 93,4 & 0,016 \\
\hline$\geq 7$ no quinto minuto & 97,6 & 98,9 & 0,007 \\
\hline Taxa de prematuridade (<37 semanas) & 12,1 & 9,8 & 0,080 \\
\hline Se o recém-nascido foi atendido por pediatra na sala de parto & 92,0 & 96,9 & $<0,001$ \\
\hline
\end{tabular}


Tabela 3

Adequação do pré-natal segundo índice proposto por Silveira et al.7* entre as gestantes residentes no município de Rio Grande, RS, 2007.

\begin{tabular}{|c|c|c|c|}
\hline \multirow[t]{3}{*}{ Variável } & \multicolumn{2}{|c|}{ Setor de saúde } & \multirow[t]{3}{*}{$p$} \\
\hline & Público ( $N=1493)$ & Privado $(\mathrm{N}=954)$ & \\
\hline & $\%$ & $\%$ & \\
\hline Adequação* do pré-natal segundo critério proposto por Silveira et al.7: & & & $<0,001$ \\
\hline Ignorado & 7,2 & 5,3 & \\
\hline Inadequado & 6,2 & 1,5 & \\
\hline Intermediário & 68,2 & 74,7 & \\
\hline Adequado & 18,4 & 18,5 & \\
\hline
\end{tabular}

* Adequado: seis ou mais consultas; início do pré-natal antes de cinco meses de gestação e mínimo de dois registros dos três exames (urina, VDRL e hemoglobina); inadequado: três consultas; início do pré-natal após o sétimo mês de gestação e não realização de qualquer exames laboratorial do item anterior; intermediário: para as demais situações.

\section{Discussão}

A assistência ao pré-natal e ao parto no município de Rio Grande mostrou-se significativamente melhor entre gestantes atendidas no setor privado no que diz respeito ao número de consultas e de início do prénatal, cobertura antitetânica, dosagem de hemoglobina, realização de ultrassonografia obstétrica, e demais exames clínicos de rotina. Além disso, estas gestantes tiveram a pressão arterial mais frequentemente verificada, seu parto foi mais comumente realizado por médico, apresentaram menor ocorrência de natimorto e seus filhos melhor índice de Apgar no primeiro e no quinto minuto e foram ainda mais comumente atendidos na sala de parto por pediatras.

Gestantes atendidas no setor público apresentaram melhor desempenho no que se refere à pesagem, verificação da altura uterina, realização de exame comum de urina, VDRL e suplementação com sulfato ferroso. Foram ainda menos frequentemente submetidas à episiotomia e cesariana. Não houve diferença significativa entre os dois grupos no que se refere à prevalência de baixo peso ao nascer, prematuridade, utilização de fórcipe e segundo o índice de adequação do pré-natal proposto por Silveira et al. 7

Ao interpretar estes resultados há que lembrar que como se trata de um estudo transversal, seu objetivo foi apenas mostrar a prevalência de desfechos para diversas variáveis relativas ao pré-natal entre o setor público e o privado, e que os indicadores apresentados refletem o período da coleta de dados. Vale destacar ainda que diversas outras variáveis relacionadas ao comportamento da gestante bem como aquelas associadas à ocorrência de eventos desfa- voráveis em gravidezes anteriores, que sabidamente influenciam a busca pelos serviços de pré-natal, e que, portanto, têm repercussão sobre o índice de adequação avaliado não foram consideradas.

A cobertura de pré-natal observada para o município de Rio Grande é, pelo menos, igual àquela encontrada em outros municípios brasileiros 3,11 e superior à encontrada para outras localidades menores ou para o país como um todo. ${ }^{12,13}$ Isto vale também para o número de consultas realizadas, idade em que foram iniciadas, e número de exames clínicos e laboratoriais realizados.11-13

Os resultados aqui obtidos confirmam vantagem sistemática e significativa para gestantes atendidas no setor privado em relação ao setor público. Isto se deve, possivelmente, ao fato de possuírem melhor nível socioeconômico, o que possibilitou a compra de serviços e a busca por cuidados, sobretudo para àqueles mais conhecidos como, por exemplo, ultrassonografia obstétrica.

Chama a atenção o fato de o exame comum de urina e testagem para sífilis (VDRL) terem alcançado índices tão baixos de cobertura, sobretudo no setor privado, sendo, inclusive, inferior ao observado no setor público. Era de se esperar o oposto, visto que gestantes do setor privado apresentam melhor nível de escolaridade e renda. ${ }^{5,14}$ Em relação ao VDRL, o argumento de que, ao realizar exame de sangue, a mãe não sabe para quais doenças será realizado o teste, pode auxiliar na explicação da baixa cobertura para ambos os grupos, mas não justifica a diferença observada entre eles. A realização de VDRL e de exame comum de urina permite identificar precocemente sífilis e infecção do tato urinário, respectivamente. Estas doenças encontram-se fortemente associadas a eventos desfavoráveis durante a 
gestação e o parto tais como abortamento, baixo peso ao nascer, retardo de crescimento intrauterino e, por conseguinte, maior morbimortalidade infantil. 15

A suplementação com sulfato ferroso foi baixa nos dois grupos, sendo mais baixa ainda entre gestantes atendidas no setor privado. Todas as gestantes deveriam receber sulfato ferroso em dose diária a partir da vigésima semana de gestação a fim de prevenir entre elas a ocorrência de hemorragias, prematuridade, abortamento, fadiga, e nos seus filhos, retardo de crescimento, desenvolvimento e baixa imunidade, entre outros. 16

A ocorrência de cesariana é abusiva no Brasil. Quase metade de todos os partos no país ocorre por cesariana. Este índice é cerca de três vezes superior ao recomendado pela Organização Mundial da Saúde. E a tendência de crescimento vem se consolidando nos últimos anos. Segundo o SINASC (Sistema de Informações de Nascidos Vivos), a taxa de cesarianas no país aumentou de 39\% em 1994 para 44\% em 2005.13 Em Rio Grande, a taxa de cesariana já ultrapassa metade de todos os partos (52\%). No setor privado, a prevalência de cesarianas é $63 \%$ maior que no setor público ( $70 \%$ vs $43 \%$ ). Isto pode ser atribuído muito mais a diferentes preferências de médicos e parturientes do que propriamente de condições clínicas adversas. ${ }^{14}$ Estudo conduzido em Pelotas mostrou que a ocorrência de cesariana é substancialmente maior entre mães de menor risco gestacional.11 A tendência é que, entre aquelas do setor privado, em o parto ocorrendo em ambiente hospitalar e havendo tempo hábil para intervenção médica, atinja-se a universalização deste procedimento. 13

A episiotomia é outra intervenção realizada em demasia. A sua utilização de forma rotineira, mesmo sendo entre as mulheres de menor idade, primíparas, dentre outras condições, não se justifica do ponto de vista científico. Pelo menos sete e oito gestantes em cada dez foram submetidas a este procedimento nos setores público e privado, respectivamente. Este percentual é muito semelhante ao observado para o Brasil como um todo (72\%), variando de $55 \%$ na Região Norte a $80 \%$ na Região Sudeste. ${ }^{13}$ Não há um índice ótimo determinado para realização deste procedimento, mas extenso estudo de revisão mostrou que não há razão para que a taxa de ocorrência de episiotomia exceda $30 \%$ dos partos. ${ }^{17}$

A utilização do índice proposto por Silveira et $a l .,{ }^{7}$ mais abrangente por levar em conta a realização de exames laboratoriais, estes tidos como indicadores da qualidade do pré-natal, mostrou que não houve diferença entre os grupos estudados. Isto ajuda talvez a explicar a ausência de impacto significativo em desfechos como baixo peso ao nascer, prematuridade, entre outros, em ambos os grupos. No entanto, estes aspectos precisam ser estudados em maior profundidade, do contrário a ocorrência de desfechos desfavoráveis muito pouco se modificará em qualquer tipo de gestante.

Em termos de recomendação, sobretudo, ao setor público, fica evidente a necessidade de aumentar o percentual de gestantes que: a) realizam seis ou mais consultas; b) iniciam o pré-natal no primeiro trimestre; c) recebem imunização antitetânica completa; d) realizam exame comum de urina, sífilis, HIV, exame ginecológico, mamas e citopatológico de colo uterino; e) seus filhos, de receberem atendimento de pediatra ainda na sala de parto. Por fim, há necessidade de aumentar a oferta de sulfato ferroso, principalmente para gestantes do setor privado e de, sobretudo entre estas, reduzir a taxa de cesarianas e episiotomia, intervenções claramente realizadas em demasia.

\section{Referências}

1. Victora CG. Intervenções para reduzir a mortalidade infantil pré-escolar e materna no Brasil. Rev Bras Epidemiol. 2001; 4: 3-69.

2. Brasil. Ministério da Saúde. Secretaria de Políticas de Saúde. Área Técnica da Mulher. Programa de Humanização no Pré-natal e Nascimento. Rev Bras Saúde Matern Infant 2002; 2 : 69-71.

3. Coimbra L, Figueiredo FP, Silva AAM, Barbieri MA, Bettiol H, Caldas AJM, Mochel EG, Ribeiro VS. Inadequate utilization of prenatal care in two Brazilian birth cohorts. 2007; Braz J Med Biol Res. 40: 1195-202.

4. Coimbra L, Silva AAM, Mochel EG, Alves MTSSB, Ribeiro VS, Aragão VMF, Bettiol H. Fatores associados à inadequação do uso da assistência pré-natal. Rev Saúde Pública. 2003; 37: 456-62.

5. Nascimento LFC. Perfil de gestantes atendidas nos períodos pré-natal e perinatal: estudo comparativo entre os serviços público e privado em Guaratinguetá, São Paulo. Rev Bras Saúde Matern Infant. 2003; 3: 187-94.

6. Takeda S. Avaliação da qualidade do pré-natal: Programa para Gestantes em Unidade de Atenção à Saúde [dissertação]. Pelotas: Universidade Federal de Pelotas; 1993.

7. Silveira DS, Santos IS, Costa JSD. Atenção pré-natal na rede básica: uma avaliação da estrutura e do processo. Cad Saúde Pública. 2001; 17: 131-9. 
8. Fundação de Economia e Estatística Siegfried Emanuel Heuser. Governo do Estado do Rio Grande do Sul. [acessado em 2 fev 2010]. Disponível em: http://www.fee.rs.gov.br/feedados/consulta/menu_consultas .asp?tp_Pesquisa=var_Anual

9. Dean AG, Dean JA, Coulombier D, Brendel KA, Smith DC, Burton AH, et al. Epi-Info, Version 6: A Word Processing, Database, and Statistics Program for Epidemiology on Microcomputers. Atlanta: Centers of Disease Control and Prevention; 2002.

10. StataCorp. Stata statistical software: release 7.0. College Station: Stata Corporation; 2001.

11. Cesar JA, Matijasevich A, Santos IS, Barros AJD, Dias-daCosta JS, Barros FC, Victora CG. The use of maternal and child health services in three population-based cohorts in Southern Brazil, 1982-2004. Cad Saúde Pública 2008; 24 (Suppl 3): S427-36.

12. Chrestani MAD, Santos IS, Cesar JA, Winckler LS, Gonçalves TS, Neumann NA. Assistência à gestação e ao parto: resultados de dois estudos transversais em áreas pobres das regiões Norte e Nordeste do Brasil. Cad Saúde Pública. 2008; 24: 1609-18.

Recebido em 3 de setembro de 2010

Versão final apresentada em 15 de junho de 2011

Aprovado em 20 de julho de 2011
13. Brasil. Ministério da Saúde e Centro Brasileiro de Análise e Planejamento. Pesquisa Nacional de Demografia e Saúde da Criança e da Mulher - PNDS 2006: Dimensões do Processo Reprodutivo e da Saúde da Criança. Série G: Estatística e Informação em Saúde. Brasília, DF; 2009.

14. Mendoza-Sassi RA, Cesar JA, Silva PR, Denardin G, Mendes M. Risk factors for cesarean section by category of health service. Rev Saúde Pública. 2010; 44: 80-9.

15. Darmstadt GL, Bhutta ZA, Cousens S, Adam T, Walker N, de Bernis L. Evidence-based, cost-effective interventions: how many newborn babies can we save? Lancet. 2005; 365 : 977-88.

16. Brasil. Ministério da Saúde. Pré-natal e puerpério: atenção qualificada e humanizada. Manual técnico. Brasília, DF; 2005.

17. Carroli G, Belizan J. Episiotomy for vaginal birth. Cochrane Review. The Cochrane Library, n. 1, 2004. Chichester: Oxford: Update software. 